\title{
Sensitivity of Rapid Antigen Testing and RT-PCR Performed on Nasopharyngeal Swabs versus Saliva Samples in COVID-19 Hospitalized Patients: Results of a Prospective Comparative Trial (RESTART)
}

\author{
Antonios Kritikos ${ }^{1, *}{ }^{+}$, Giorgia Caruana ${ }^{1,+} \mathbb{D}$, René Brouillet ${ }^{1}$, John-Paul Miroz ${ }^{2}$, Samia Abed-Maillard ${ }^{2}$, \\ Geraldine Stieger $^{2}$, Onya Opota ${ }^{1}{ }^{(D}$, Antony Croxatto ${ }^{1}$, Peter Vollenweider ${ }^{3}$, Pierre-Alexandre Bart ${ }^{3}$, \\ Jean-Daniel Chiche ${ }^{2}$ and Gilbert Greub ${ }^{1, *(D)}$
}

check for updates

Citation: Kritikos, A.; Caruana, G.; Brouillet, R.; Miroz, J.-P.;

Abed-Maillard, S.; Stieger, G.; Opota, O.; Croxatto, A.; Vollenweider, P.; Bart, P.-A.; et al. Sensitivity of Rapid Antigen Testing and RT-PCR Performed on Nasopharyngeal Swabs versus Saliva Samples in COVID-19 Hospitalized Patients: Results of a Prospective Comparative Trial (RESTART). Microorganisms 2021, 9, 1910. https://doi.org/10.3390/ microorganisms 9091910

Academic Editor: Raffaele Bruno

Received: 13 August 2021

Accepted: 7 September 2021

Published: 9 September 2021

Publisher's Note: MDPI stays neutral with regard to jurisdictional claims in published maps and institutional affiliations.

Copyright: (c) 2021 by the authors. Licensee MDPI, Basel, Switzerland. This article is an open access article distributed under the terms and conditions of the Creative Commons Attribution (CC BY) license (https:/ / creativecommons.org/licenses/by/ $4.0 /)$.
1 Institute of Microbiology, Lausanne University Hospital and University of Lausanne, 1011 Lausanne, Switzerland; Giorgia.Caruana@chuv.ch (G.C.); Rene.Brouillet@chuv.ch (R.B.); Onya.Opota@chuv.ch (O.O.); Antony.Croxatto@chuv.ch (A.C.)

2 Intensive Care Unit, Lausanne University Hospital and University of Lausanne, 1011 Lausanne, Switzerland; John-Paul.Miroz@chuv.ch (J.-P.M.); Samia.Abed-Maillard@chuv.ch (S.A.-M.); Geraldine.Stieger@chuv.ch (G.S.); Jean-Daniel.Chiche@chuv.ch (J.-D.C.)

3 Service of Internal Medicine, Lausanne University Hospital and University of Lausanne, 1011 Lausanne, Switzerland; Peter.Vollenweider@chuv.ch (P.V.); Pierre-Alexandre.Bart@chuv.ch (P.-A.B.)

* Correspondence: Antonios.kritikos@chuv.ch (A.K.); Gilbert.Greub@chuv.ch (G.G.); Tel.: +41-(0)21-314-4979 (G.G.)

+ These authors contribute equally to this work.

Abstract: Saliva sampling could serve as an alternative non-invasive sample for SARS-CoV-2 diagnosis while rapid antigen tests (RATs) might help to mitigate the shortage of reagents sporadically encountered with RT-PCR. Thus, in the RESTART study we compared antigen and RT-PCR testing methods on nasopharyngeal (NP) swabs and salivary samples. We conducted a prospective observational study among COVID-19 hospitalized patients between 10 December 2020 and 1 February 2021. Paired saliva and NP samples were investigated by RT-PCR (Cobas 6800, Roche-Switzerland, Basel, Switzerland) and by two rapid antigen tests: One Step Immunoassay Exdia ${ }^{\circledR}$ COVID-19 Ag (Precision Biosensor, Daejeon, Korea) and Standard Q ${ }^{\circledR}$ COVID-19 Rapid Antigen Test (Roche-Switzerland). A total of 58 paired NP-saliva specimens were collected. A total of 32 of 58 (55\%) patients were hospitalized in the intensive care unit, and the median duration of symptoms was 11 days (IQR 5-19). NP and salivary RT-PCR exhibited sensitivity of $98 \%$ and $69 \%$ respectively, whereas the specificity of these RT-PCRs assays was $100 \%$. The NP RATs exhibited much lower diagnostic performance, with sensitivities of $35 \%$ and $41 \%$ for the Standard $Q^{\circledR}$ and Exdia ${ }^{\circledR}$ assays, respectively, when a wet-swab approach was used (i.e., when the swab was diluted in the viral transport medium (VTM) before testing). The sensitivity of the dry-swab approach was slightly better (47\%). These antigen tests exhibited very low sensitivity $(4 \%$ and $8 \%$ ) when applied to salivary swabs. Nasopharyngeal RT-PCR is the most accurate test for COVID-19 diagnosis in hospitalized patients. RT-PCR on salivary samples may be used when nasopharyngeal swabs are contraindicated. RATs are not appropriate for hospitalized patients.

Keywords: SARS-CoV-2 diagnosis; RT-PCR; rapid antigen testing; saliva; viral transport medium

\section{Introduction}

Rapid and accurate detection of SARS-CoV-2 infection in hospitalized patients is the cornerstone of prompt patient care and contact tracing. To date, nasopharyngeal (NP) swab real-time polymerase chain reaction (RT-PCR) remains the reference specimen for SARS-CoV-2 testing [1]. However, NP swabbing might expose healthcare workers to the risk of transmission during sampling and is a relatively invasive method, especially when 
considering the multiple samplings a patient will go through during a hospital stay. On the other hand, there is growing evidence advocating the role of salivary or oropharyngeal specimens as alternative non-invasive methods for SARS-CoV-2 diagnosis [2-6].

At the same time, and as a response to the growing SARS-CoV-2 pandemic and reagents shortages for rapid molecular systems, multiple rapid point-of-care tests (POCTs) have been added to the diagnostic pipeline for COVID-19 [7,8].

Little is known on the utility and diagnostic performance of the above-mentioned strategies in moderately to severely ill hospitalized patients. Current literature shows discordant results depending on the clinical setting studied, the sampling method or even whether a viral transport medium is used (VTM) [2-6,9-17].

In order to simultaneously investigate analytical (RT-PCR versus Rapid Antigen Test (RAT)) and sampling procedures (NP swab versus saliva specimen and use versus not of VTM), we conducted a prospective observational study in hospitalized patients. Our aim was to:

(1) Compare the diagnostic performance of NP RT-PCR and salivary RT-PCR;

(2) Evaluate the reliability of RATs in hospitalized patients;

(3) Compare the sensitivity of RATs performed on NP swab versus saliva in this specific population;

(4) Evaluate the impact of VTM on the diagnostic performance of RATs.

\section{Materials and Methods}

\subsection{Study Population}

We conducted a prospective observational study among SARS-CoV-2 positive patients hospitalized in our institution, a tertiary university hospital in Lausanne, Switzerland (CHUV), between 10 December 2020 and 1 February 2021. All patients were previously COVID-19 confirmed cases (via NP RT-PCR) and hospitalized either in the internal medicine ward or in the intensive care unit (ICU). Inclusion criteria were (a) positive NP RT-PCR for SARS-CoV-2 in the previous 5 days, (b) age $>18$ years old and (c) informed consent acquisition by the patient or the next of kin for patients incapable of providing informed consent. We collected no additional personal or clinical data beyond the usual information required for every SARS-CoV-2 test by the Federal Office of Public Health (FOPH) and our microbiology laboratory (age, sex, hospitalization ward, type and duration of symptoms). During the study period, the vast majority of SARS-CoV-2 infections in Switzerland was due to previously circulating wild-type strains. There were only a few sporadic cases of the B.1.1.7-Alpha variant.

\subsection{Sample Collection and Diagnostic Tests}

After informed consent acquisition, patients underwent two NP swabs (one diluted in universal VTM (UTM ${ }^{\circledR}$ Copan Diagnostics, Murrieta, CA, USA) (“Wet” approach) [17] and one non-diluted ("Dry approach")) and a saliva sample also diluted in the same VTM. Paired diluted NP and saliva samples were analyzed by RT-PCR (Cobas $6800^{\circledR}$, RocheSwitzerland, Basel, Switzerland) [18] and by two rapid antigen tests (RATs): One Step Immunoassay Exdia ${ }^{\circledR}$ COVID-19 Ag (Precision Biosensor, Daejeon, Korea) and Standard $Q^{\circledR}$ COVID-19 Rapid Antigen Test (Roche-Switzerland, Basel, Switzerland). The rapid antigen tests used in our study were chosen based on their performance in previous comparative clinical studies $[14,19]$. The non-diluted NP swab was tested with Standard $Q^{\circledR}$ COVID-19 Rapid Antigen Test (Roche-Switzerland, Basel, Switzerland) at patient's bedside in order to evaluate the effect of VTM on the diagnostic performance of RATs. Both "Wet" and "Dry" approaches are recommended by the manufacturers of both RATs used. An additional manipulation step using VTM tubes was performed for an in vitro evaluation of a possible dilution effect of VTM (see Section 2.4).

All samples were taken by two specialists in infectious diseases (A.K. and G.C.) or a member of a paramedic team trained in NP swabs collection (coming from a team performing all COVID-19 samples in our hospital). NP was performed according to the 
recent CDC and WHO guidelines [20,21], and the saliva sampling protocol was based on previously published data adapted for hospitalized patients (Figure S1) [5]. Each nasopharyngeal swab was performed on a different naris, which was randomly chosen for each sample.

\subsection{Quantitative SARS-CoV-2 PCR and RAT}

RT-PCR was performed in our microbiology laboratory using the automated Cobas $680{ }^{\circledR}$ system (Roche-Switzerland) [18]. In order to quantify the viral load (VL) based on the number of cycles threshold $(\mathrm{Ct})$ obtained with the molecular platform, we used the following equation, derived from RNA quantification: $\mathrm{VL}=\left(10^{\wedge}((\mathrm{Ct}-40.856) /-3.697)\right) \times 100$. Details on the methods used to derive this equation were described elsewhere [22]. The analytical limit of detection was determined to be at 1000 copies per mL. For graphical representation purposes, NP or salivary samples with undetectable VL are represented in graphs, with VL determined to be at 500 copies $/ \mathrm{mL}$.

NP swabs and saliva samples were used to assess RAT performance. NP swabs were either directly suspended in the buffer solution ("Dry approach"), or initially suspended in $3 \mathrm{~mL}$ of VTM ("Wet" approach). One hundred and fifty microliters of the sample was subsequently mixed with the buffer solution. Saliva samples were only treated with the "Wet" approach. Reading of the results was performed after 15 to $30 \mathrm{~min}$, as specified by manufacturer's instructions (on a band of immunochromatography paper for Standard $Q^{\circledR}$ COVID-19 Rapid Antigen Test or using Exdia TRF Biosensor to detect immunofluorescence signal for One Step Immunoassay Exdia ${ }^{\circledR}$ COVID-19 Ag). In case of doubtful results, the Standard $Q^{\circledR}$ COVID-19 Rapid Antigen Test test was read independently by a second person.

\subsection{In Vitro Testing of Dilution Effect}

In order to test in vitro a possible dilution effect generated by the use of VTM tubes instead of direct testing, we simulated the two sampling scenarios: the "Wet" scenario versus the "Dry" swab scenario (Figure S2). A SARS-CoV-2 positive clinical NP sample was diluted 7 times, and the series was used as internal reference for the limit of detection. Each initial sample of this dilution series represented a "Dry" swab. Then, two clean swabs were soaked in each one of the dry samples and then suspended in two further VTM tubes, thus simulating two series of "Wet" samples. Each sample from every series was tested as follows:

(i) One Step Immunoassay Exdia ${ }^{\circledR}$ COVID-19 Ag (Precision Biosensor, Daejeon, Korea) and Standard Q ${ }^{\circledR}$ COVID-19 Rapid Antigen Test (Roche-Switzerland, Basel, Switzerland) were performed after mixing $350 \mu \mathrm{L}$ of VTM with the buffer solution provided in the RAT kit;

(ii) A clean swab was first inoculated in the VTM, then mixed with the buffer solution (according to manufacturer instructions) and tested with the previously mentioned commercial kits; and

(iii) An amount of $300 \mu \mathrm{L}$ of VTM was used for RT-PCR analysis.

\subsection{Statistical Analysis}

Descriptive statistics are presented as number and percentage for categorical variables and mean \pm standard deviation (SD) or median (interquartile range; IQR) for continuous variables. Chi-square or Fisher's exact test was used for categorical variables and Wilcoxon matched-pairs rank test for continuous variables where appropriate. Sensitivity, specificity, positive and negative predictive values and $95 \% \mathrm{CI}$ were calculated to assess diagnostic performance using a positive NP or saliva RT-PCR as reference standard. All statistical analyses were performed using GraphPad Prism version 8.3.0 for Windows (GraphPad Software, San Diego, CA, USA). 


\subsection{Ethics}

This project was conducted in accordance with the Declaration of Helsinki, the principles of Good Clinical Practice and the Swiss Human Research Act (HRO). The project received approval from the Ethics Committee of canton Vaud, Switzerland (Project-ID: 2020-02818) the 4 December 2020. The study was registered on ClinicalTrials.gov with the number NCT04839094.

\section{Results}

\subsection{Patients' Characteristics}

All patients with confirmed SARS-CoV-2 infection by NP RT-PCR and admitted in the ICU or internal medicine ward during the study period were screened for eligibility criteria. Figure 1 shows the flowchart of the screening process. A total of 58 paired NP and saliva samples were performed, with a positivity rate of $85 \%$ (time elapsed from screening sample to inclusion could be up to 5 days). Baseline demographics and clinical characteristics are shown in Table 1 . Patients were predominantly males $(n=45,77 \%)$, with a median age of 70 years old (IQR 61-77). Most of them still had symptoms upon sampling ( $n=49$, $84 \%$ ), with a median duration of symptoms of 11 days (IQR, 5-19). Common symptoms on presentation were dyspnea $(n=27,46 \%)$, cough $(n=19,33 \%)$ and fever $(n=10,17 \%)$. SARS-CoV-2 VL in NP swab ranged from 3800 to $9,900,000$ copies/mL (median value 48,000 copies/mL).

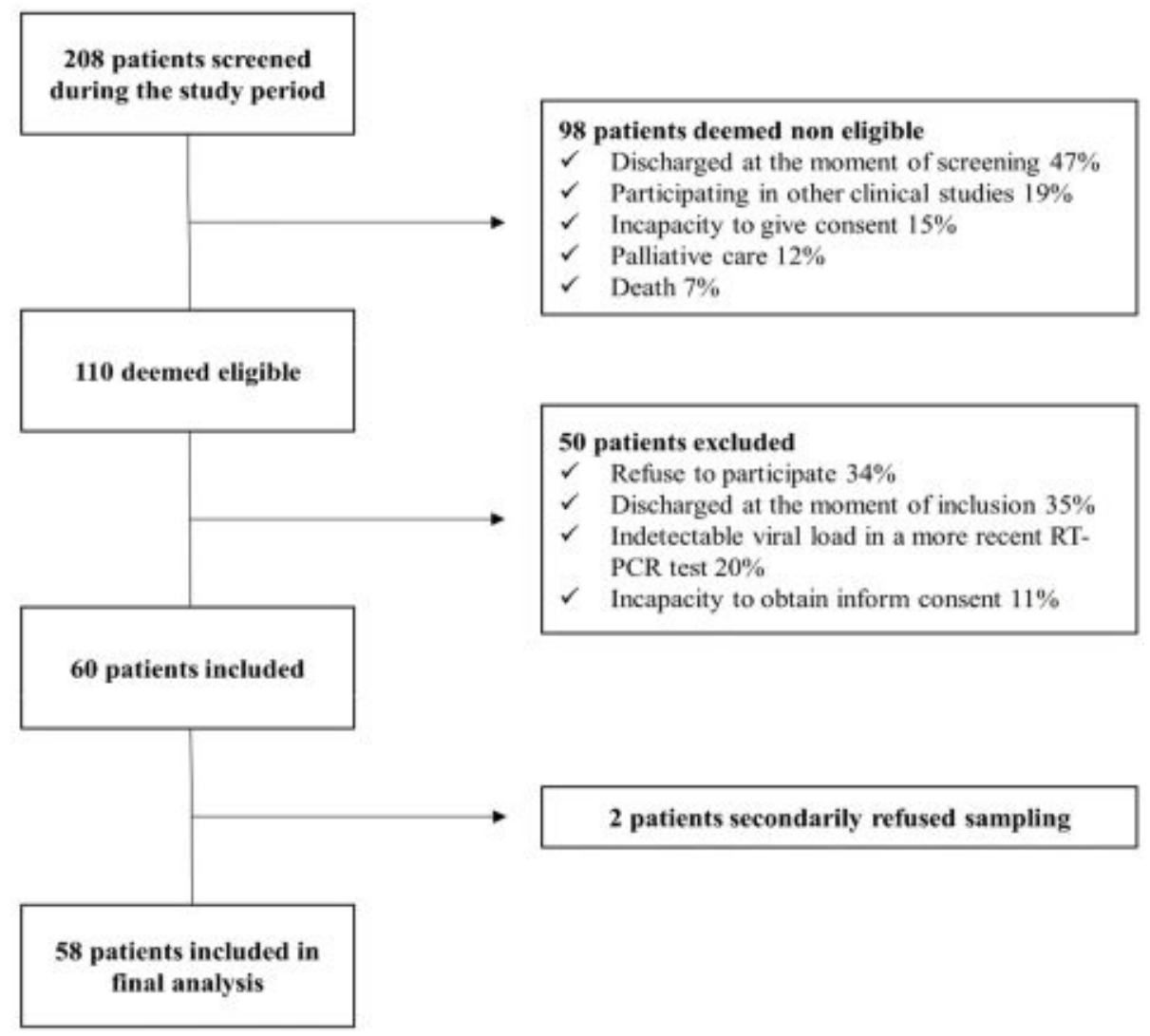

Figure 1. Flowchart of screening process. 
Table 1. Baseline demographics and clinical characteristics of patients.

\begin{tabular}{cccc}
\hline Characteristics & $\begin{array}{c}\text { Internal Medicine Patients } \\
(\boldsymbol{n}=\mathbf{2 6})\end{array}$ & $\begin{array}{c}\text { ICU Patients } \\
(\boldsymbol{n}=\mathbf{3 2})\end{array}$ & $\begin{array}{c}\text { Overall } \\
(\boldsymbol{n}=\mathbf{5 8})\end{array}$ \\
\hline $\begin{array}{c}\text { Age (y), median (IQR) } \\
\text { Male, } \boldsymbol{n}(\mathbf{\%})\end{array}$ & $70(61-77)$ & $71(62-78)$ & $70(61-77)$ \\
$\begin{array}{c}\text { Patients with symptoms } \\
\text { upon sampling, } \boldsymbol{n}(\mathbf{\%})\end{array}$ & $20(77)$ & $25(78)$ & $45(77)$ \\
$\begin{array}{c}\text { Duration of symptoms, } \\
\text { median (IQR) }\end{array}$ & $21(81)$ & $28(87)$ & $49(84)$ \\
Type of symptoms, $\boldsymbol{n}(\mathbf{\%})$ & $11(5-19)$ & $11(6-19)$ & $11(5-19)$ \\
Fever & & & \\
Cough & $5(19)$ & $5(16)$ & $10(17)$ \\
Dyspnea & $8(31)$ & $11(34)$ & $19(33)$ \\
Fatigue & $7(27)$ & $20(62)$ & $27(46)$ \\
Anosmia/Dysgeusia & $4(15)$ & $3(9)$ & $7(12)$ \\
Other & $1(4)$ & $2(6)$ & $3(5)$ \\
Viral load, median & $6(23)$ & $8(25)$ & $14(24)$ \\
(IQR) & 43,000 & 82,000 & 48,000 \\
& $(3700-9,100,000)$ & $(4500-12,000,000)$ & $(3800-$ \\
& & & $9,900,000)$ \\
\hline
\end{tabular}

\subsection{Diagnostic Performance of RT-PCR (NP versus Saliva) and Rapid Antigen Testing}

The diagnostic performance of RT-PCR and RAT for diagnosis of SARS-CoV-2 infection is shown on Table 2. A positive NP RT-PCR or salivary RT-PCR was selected as the reference standard comparator.

Table 2. Overall diagnostic performance metrics of the different diagnostic approaches used.

\begin{tabular}{|c|c|c|c|c|c|}
\hline \multicolumn{2}{|c|}{ Diagnostic Test } & $\begin{array}{c}\text { Sensitivity (\%) } \\
(95 \% \mathrm{CI})\end{array}$ & $\begin{array}{c}\text { Specificity (\%) } \\
\text { (95\% CI) }\end{array}$ & $\begin{array}{l}\text { PPV (\%) } \\
(95 \% \text { CI) }\end{array}$ & $\begin{array}{l}\text { NPV (\%) } \\
(95 \% \text { CI) }\end{array}$ \\
\hline \multicolumn{2}{|c|}{ Nasopharyngeal RT-PCR } & $98(89-100)$ & $100(66-100)$ & $100(93-100)$ & $90(56-98)$ \\
\hline \multicolumn{2}{|c|}{ Salivary RT-PCR PCR } & $69(55-82)$ & $100(66-100)$ & $100(90-100)$ & $37.5(28-48)$ \\
\hline \multirow{3}{*}{$\begin{array}{l}\text { Nasopharyngeal } \\
\text { rapid antigen test }\end{array}$} & Exdia $^{\circledR}($ ("Wet") & $41(27-56)$ & $100(63-100)$ & $100(83-100)$ & $22(18-26)$ \\
\hline & Roche $^{\circledR}($ ("Wet") & $35(22-50)$ & $100(66-100)$ & $100(82-100)$ & $22(19-26)$ \\
\hline & Roche $^{\circledR}$ ("Dry") & $47(35-62)$ & $100(66-100)$ & $100(86-100)$ & $26(21-31)$ \\
\hline \multirow{2}{*}{$\begin{array}{l}\text { Salivary rapid } \\
\text { antigen test }\end{array}$} & $\operatorname{Exdia}^{\circledR}($ (Wet") & $8(2-20)$ & $100(66-100)$ & $100(51-100)$ & $17(15-18)$ \\
\hline & Roche $^{\circledR}($ ("Wet") & $4(0.5-14)$ & $100(66-100)$ & $100(18-100)$ & $16(15-17)$ \\
\hline
\end{tabular}

A positive nasopharyngeal or salivary RT-PCR was used as reference standard. "Wet" and "Dry" rapid antigen tests refer to testing performed after soak of the swab ("Wet") in universal viral transport medium (Copan Italia UTM-RT) or directly to patient's bedside after sampling ("Dry").

NP and salivary RT-PCR exhibited an overall sensitivity of $98 \%$ and $69 \%$, respectively, whereas the specificity of both assays was of $100 \%$. Noteworthy, the sensitivity of salivary PCR increased to $81 \%$ (95\% CI: 59-88) in patients presented with a duration of symptoms of less than 10 days. VL (copies/mL) in NP swabs was significantly higher than that detected on salivary specimens for up to 20 days after illness onset (Figure 2). Median VL value in positive NP swabs with negative paired saliva specimens was 3700 copies $/ \mathrm{mL}$ (IQR, 2900-9675) (Figure 2). Median duration of illness for those patients was 15 days (IQR, 9-21). ICU patients had higher VL compared with patients hospitalized in the internal medicine ward when tested either by NP or saliva RT-PCR (Figure 3). Pair testing results are shown in Figure 3. An analysis of the agreement between the two specimens (NP versus saliva) revealed a fair agreement, with a kappa coefficient of 0.37 (95\% CI $0.16-0.59 ; p=0.001)$ and an overall proportion of agreement of $72 \%$ (proportion of positive agreement $80 \%$ and proportion of negative agreement 53\%) (Figure S3). 


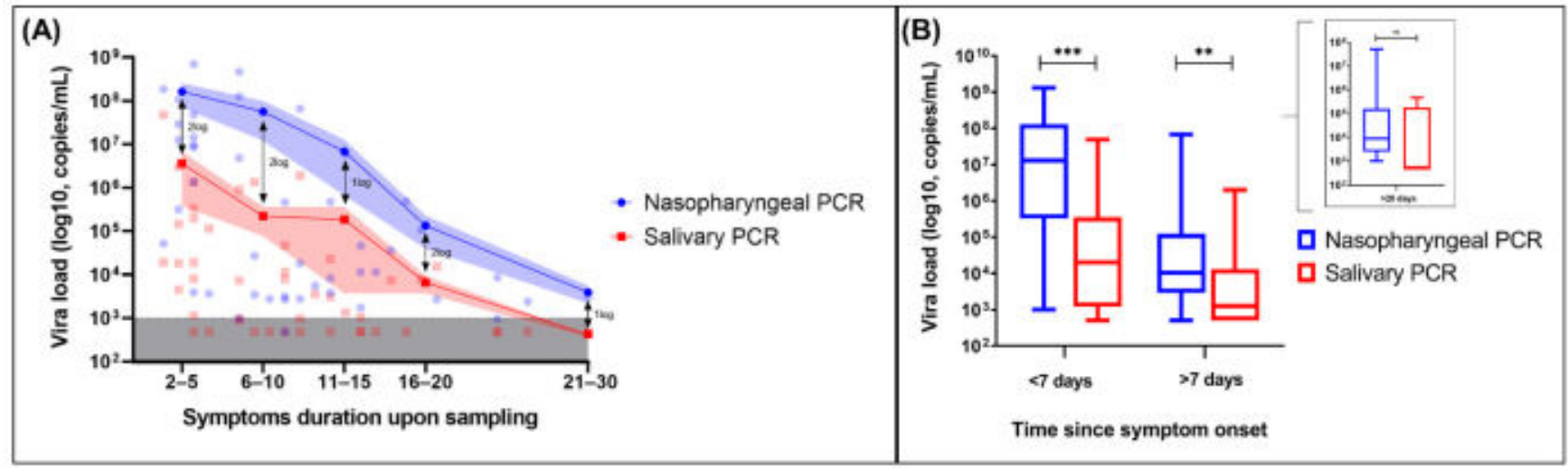

Figure 2. Viral load dynamics of NP RT-PCR versus saliva RT-PCR. (A) Kinetics of nasopharyngeal and salivary viral load according to symptom duration upon sampling. The lines connect the mean values of each period, and the shaded areas indicate the mean standard errors (SEM). The inferior grey shaded area represents the detection limit (1000 copies/mL). Specimens with undetectable viral load are shown within the grey dashed area. (B) Box and whisker plots comparing viral load between nasopharyngeal and salivary PCR on different periods since symptom onset. Boxes extend from 25 th to 75 th percentiles and whiskers show 5th and 95th percentiles. The lines in the middle of the boxes are plotted at median values. (Results were compared using Wilcoxon matched-pairs nonparametric test, ${ }^{* * *}=p<0.001,{ }^{* *}=p<0.01$, ns $=$ non-significant).

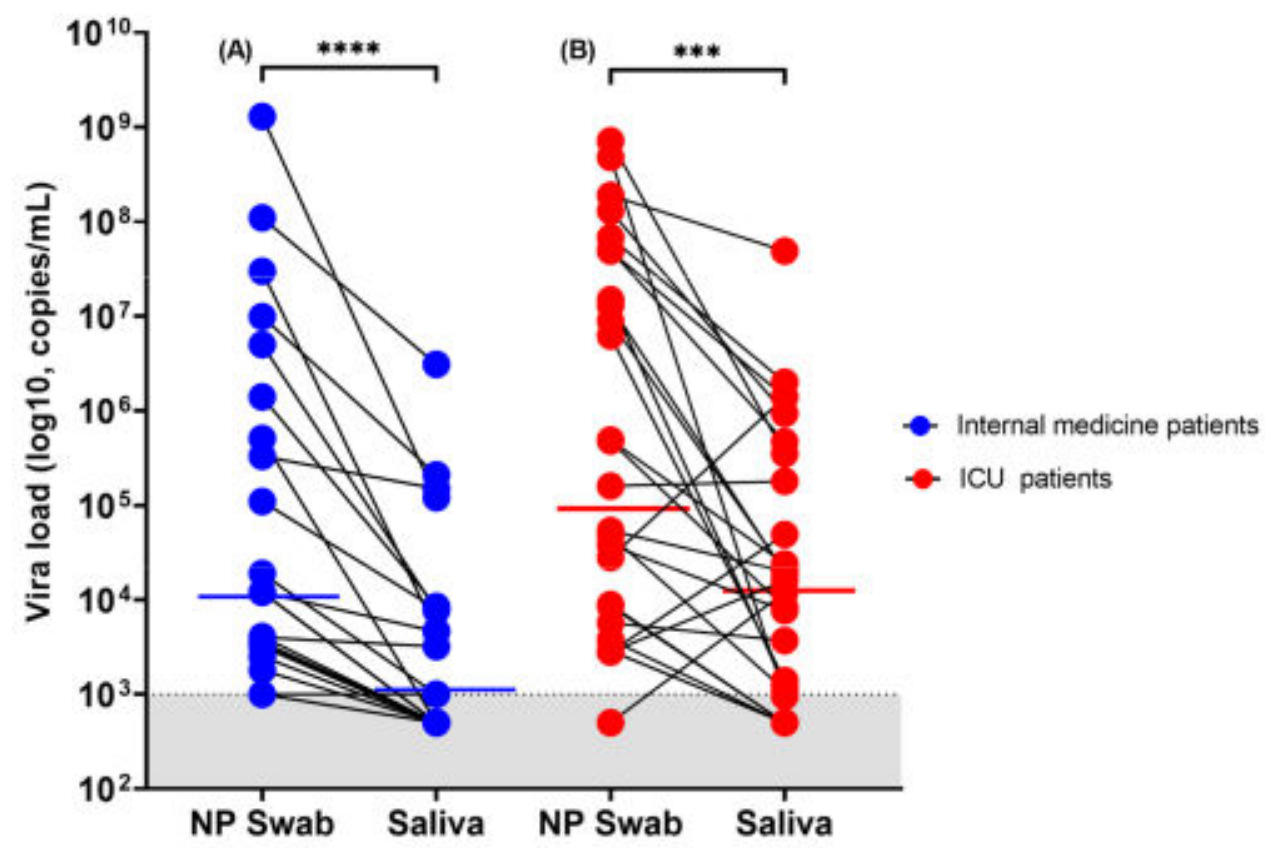

Figure 3. Viral load trend in person-matched NP and saliva samples $(n=49)$. (A) Patients hospitalized in the internal medicine ward and (B) patients hospitalized in the ICU. The dotted line and shaded grey area delimit our assay's detection limit. For graphical representation purposes, samples within the undetectable area are represented with values determined to be at 500 copies $/ \mathrm{mL}$. The red and blue horizontal lines represent median values ${ }^{* * * *}=p<0.0001$ and ${ }^{* * *}=p<0.001$ with Wilcoxon signed-rank test).

RATs exhibited much lower diagnostic performance, with sensitivities of $41 \%$ and $35 \%$ for the Exdia ${ }^{\circledR}$ and Standard $Q^{\circledR}$ assays, respectively, among hospitalized patients when a wet-swab approach was used (Table 2). Interestingly, the sensitivity of the dry-swab approach was slightly better (sensitivity $47 \%$ (95\% CI: 35-62) for Standard $Q^{\circledR}$ assay). These antigen tests exhibited very low sensitivity of $8 \%$ and $4 \%$ for Exdia ${ }^{\circledR}$ and Standard $Q^{\circledR}$ assays, respectively, when applied to salivary swabs. Figure 4 shows RAT results according to illness duration and VL. All RATs performed better in high VL or early in the course of the disease (especially if VL $>10^{6}$ copies / $\mathrm{mL}$ and illness duration $<10$ days) (Figure S4). 


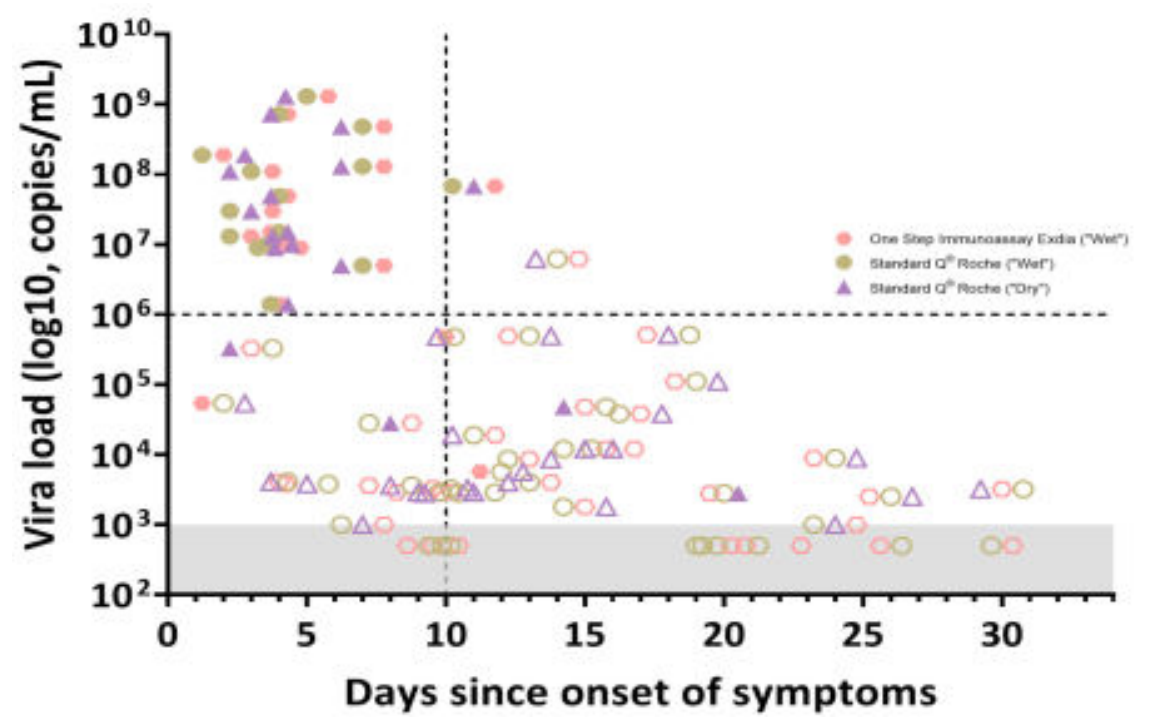

Figure 4. Rapid antigen test results according to time since onset of symptoms and viral load. Full shaded symbols show positive results while no shaded symbols show negative RAT results.

\subsection{In Vitro Dilution Effect}

The limit of detection for Standard ${ }^{\circledR}$ COVID-19 Rapid Antigen Test (Roche-Switzerland) varied between $1.9 \times 10^{6}$ and $4.3 \times 10^{5}$ copies $/ \mathrm{mL}$ in the dry and wet procedures, respectively, while for Exdia ${ }^{\circledR}$ it varied between $1.1 \times 10^{7}$ and $2.3 \times 10^{6}$ copies $/ \mathrm{mL}$, respectively, considering the gene E detected by Cobas $6800^{\circledR}$ (Table S1). Quantification of the VL showed lower $\mathrm{Ct}$ values (between 2 and $5 \mathrm{Ct}$, corresponding to a difference of 1-2 logs) for the dry series compared with the respective wet series. Ct results from the E or the RdRP gene were comparable, demonstrating a limited intra-method variability.

\section{Discussion}

The present study sought to evaluate the role of alternative and non-invasive methods for diagnosis of SARS-CoV-2 infection in moderately and critically ill-hospitalized patients. Moreover, to our knowledge this is the first study to evaluate the impact of VTM on RAT results for SARS-CoV-2 diagnosis.

Saliva sampling is a promising alternative to NP swabs for SARS-CoV-2 diagnosis, given the ease of collection and comfort for repetitive testing as well as highly reliable results [2-6,9-12]. Nonetheless, only a few studies evaluated the diagnostic performance of saliva as compared with NP swab in hospitalized patients [3,11-13], with results being conflicting. Our study evaluated hospitalized patients with moderate to very severe disease. Most of them were still symptomatic upon sampling $(84 \%)$ and had a wide distribution of VL, ranging from $10^{3}$ to $10^{9}$ copies/mL. Overall, NP RT-PCR was more sensitive in diagnosis of SARS-CoV-2 infection than RT-PCR performed in saliva sample. VL detected in NP swab was higher (1-2 log copies/mL) than in saliva specimens. It is noteworthy, however, that the sensitivity of salivary RT-PCR increased considerably (from $69 \%$ to $81 \%$ ) when considering patients presenting early in the course of the disease ( $<10$ days). Progressive decrease in VL over time [14] might explain the loss of sensitivity of the salivary RT-PCR, which is more pronounced when testing patients with more than 10 days of symptoms. In fact, patients in our study with a positive NP swab and negative saliva sample had low VL (median value of 3700 copies $/ \mathrm{mL}$ ) and a median duration of symptoms of 15 days. This raises the question of the infectivity of those patients and suggest that patients not detected by salivary RT-PCR are those who have low infectivity potential and are late presenters in the course of the disease. Therefore, the results of our study, along with previous published data $[3,9,11-13]$, suggest that saliva specimens could be a fair noninvasive alternative to NP swabs (if NP swab is contraindicated for example), especially for those presenting early in the course of illness. Recently published data furthermore 
advocate the role of combined oropharyngeal and anterior nasal or mid-turbinate swabs as alternative diagnostic methods that are amendable to patient self-collection [23-25].

A second goal of our study was to evaluate the diagnostic performance of RATs among hospitalized patients. RATs exhibited unacceptable overall diagnostic performance for hospitalized patients whether performed in NP swab or in saliva specimens. To date, both the Federal Office of Public health in Switzerland and the Swiss Society of Microbiology recommend RATs only within the first four days of symptoms [26]. Nevertheless, very few (if any) hospitalized patients present within this timeframe since the onset of symptoms. When we tested the diagnostic performance of RAT for patients presenting with less than 10 days of illness, sensitivity remained very low. RATs yielded high diagnostic performance only for patients with high VL ( $\geq 10^{6}$ copies/mL) (Figure S4). The much lower diagnostic performance of RATs in saliva might be explained by the lower VL in saliva as compared to NP swabs or eventually the presence of mucosal secretory immunoglobulins targeting SARS-CoV-2 antigens and thus competing with a RAT for the same target [14,27].

Finally, our study evaluated the role of VTM in SARS-CoV-2 diagnosis by RAT. While both "Wet" and "Dry" procedures are recommended by the manufacturer, previous data suggest that VTM can influence the diagnostic performance of RT-PCR and RAT for SARS-CoV-2 detection $[15,28]$. This is the first study to our knowledge that evaluates "head-to-head" a "Wet" versus "Dry" RAT procedure for SARS-CoV-2 detection. Our in vitro evaluation showed higher Ct levels for the "Wet" series of both antigen tests used, suggesting a dilution effect when the swab is immerged in the VTM. Our head-to-head clinical comparison confirmed the in vitro experimentation, showing that the "Dry" NP swab performed slightly better than the "Wet" one (sensitivity increasing from 35\% to 47\%), likely due to a decreased dilution of the sample. While RATs cannot be recommended for hospitalized patients, the observed difference between "Wet" and "Dry" swabs should be taken into account when performing a RAT for SARS-CoV-2 diagnosis in an outpatient setting. Still, when RATs are just used as a supplementary triaging step at the hospital entry to hasten isolation of highly contagious patients, the use of a wet swab approach is also acceptable since the lower sensitivity is compensated by the fact that in a high-risk pandemic period, performing a single sampling for both RAT and PCR is likely a good and effective option [14].

Our study had the strength to prospectively test hospitalized patients with variable disease severity (ICU versus internal medicine ward) and a wide distribution of VL and symptom duration. This study provides insight into the diagnostic performance of different tests under real-life conditions in hospitalized patients. Moreover, it is the first study to our knowledge, to evaluate the impact of VTM on the RATs' ability to detect SARS-CoV-2.

On the other hand, this study has a few limitations as well. Its monocentric nature and limited sample size require our results to be confirmed by larger prospective trials. Hospitalized patients may have altered saliva production or composition [29] that could influence saliva-based diagnostic strategies or even explain the differences observed in salivary RT-PCR performance among severely and mildly ill COVID-19 patients. We chose to use a validated and easy-to-use non-invasive saliva collection procedure [5]. It is possible that other methods of saliva collection (such as throat washing for example) would have improved diagnostic yield and should therefore be tested in other comparative trials. Some examples of methods currently under evaluation include, for example, collection devices that use colorimetric indicators to tell a patient when enough specimen has been obtained [25]. Future studies might also consider using a composite gold standard consisting of multiple site sampling to try to improve the reference standard. Finally, in order to fully understand SARS-CoV-2 viral dynamics, studies will have to obtain multiple specimens, ideally from the same patient, in a longitudinal design.

In conclusion, our study shows that NP swab RT-PCR was the most sensitive method to diagnose SARS-CoV-2 infection in moderately to critically ill hospitalized patients. Salivary RT-PCR could be used as an alternative non-invasive method if NP swabs are 
contraindicated, particularly for patients presenting early in the course of the disease. A VTM-induced dilution effect can impact diagnostic performance of RATs.

Supplementary Materials: The following are available online at https:/ /www.mdpi.com/article/10 .3390 / microorganisms9091910/s1, Figure S1: Procedure for collecting saliva for COVID-19 saliva test, Figure S2: In vitro experiment of the dilution effect for "Dry" versus "Wet" swab procedure, Figure S3: Agreement matrix of matched NP swab and saliva specimens, Figure S4: RAT sensitivity after stratification for viral load and duration of symptoms, Table S1: Limit of detection for "dry" versus "wet" swab procedure during the in vitro simulation experiment.

Author Contributions: A.K., G.C. and G.G. conceived and designed the study, performed the literature research, analyzed data and performed statistical analyses, drafted and revised the article. Moreover, A.K. and G.C. collected patient data and performed hands-on conduct of the study's diagnostic procedures. R.B. conceived and designed the study, provided essential reagents and materials and performed in vitro experiments. J.-P.M. was responsible for project administration in the ICU and collected patient data. S.A.-M. and G.S. were responsible for project administration in the ICU. O.O. designed the study and provided essential reagents and materials. A.C. conceived and designed the study and provided essential reagents and materials. P.V. designed the study and was responsible for project administration in the internal medicine ward. P.-A.B. designed the study and was responsible for project administration in the internal medicine ward. J.-D.C. designed the study and was responsible for project administration in the ICU. All authors have read and agreed to the published version of the manuscript.

Funding: This research received no external funding.

Institutional Review Board Statement: The study was conducted according to the guidelines of the Declaration of Helsinki, and approved by the Ethics Committee of Canton Vaud, Switzerland (protocol code 2020-02818, approved the 04.12.2020).

Informed Consent Statement: Informed consent was obtained from all subjects involved in the study.

Data Availability Statement: Data supporting reported results will be available upon request for the peer-review process.

Acknowledgments: We would like to thank all the technical staff of the Microbiology Institute of Lausanne University Hospital who performed routine RT-PCRs as well as the entire medical and nursing team of the intensive care unit and internal medicine ward for their precious help in the realization of the project.

Conflicts of Interest: The authors declare no conflict of interest.

\section{References}

1. WHO. Diagnostic Testing for SARS-CoV-2. Available online: https://www.who.int/publications/i/item/diagnostic-testing-forsars-cov-2 (accessed on 7 April 2021).

2. Butler-Laporte, G.; Lawandi, A.; Schiller, I.; Yao, M.; Dendukuri, N.; McDonald, E.G.; Lee, T.C. Comparison of Saliva and Nasopharyngeal Swab Nucleic Acid Amplification Testing for Detection of SARS-CoV-2: A Systematic Review and Meta-analysis. JAMA Intern. Med. 2021, 181, 353-358. [CrossRef] [PubMed]

3. Jamal, A.J.; Mozafarihashjin, M.; Coomes, E.; Powis, J.; Li, A.X.; Paterson, A.; Anceva-Sami, S.; Barati, S.; Crowl, G.; Faheem, A.; et al. Sensitivity of nasopharyngeal swabs and saliva for the detection of severe acute respiratory syndrome coronavirus 2 (SARS-CoV-2). Clin. Infect. Dis. 2020, 72, 1064-1066. [CrossRef] [PubMed]

4. Pasomsub, E.; Watcharananan, S.P.; Boonyawat, K.; Janchompoo, P.; Wongtabtim, G.; Suksuwan, W.; Sungkanuparph, S.; Phuphuakrat, A. Saliva sample as a non-invasive specimen for the diagnosis of coronavirus disease 2019: A cross-sectional study. Clin. Microbiol. Infect. 2021, 27, 285.e1-285.e4. [CrossRef] [PubMed]

5. Schwob, J.-M.; Miauton, A.; Petrovic, D.; Perdrix, J.; Senn, N.; Jaton, K.; Opota, O.; Maillard, A.; Minghelli, G.; Cornuz, J.; et al. Antigen rapid tests, nasopharyngeal PCR and saliva PCR to detect SARS-CoV-2: A prospective comparative clinical trial. medXriv 2020. Available online: https:/ / www.medrxiv.org/content/10.1101/2020.11.23.20237057v1 (accessed on 7 April 2021).

6. Huber, M.; Schreiber, P.W.; Scheier, T.; Audigé, A.; Buonomano, R.; Rudiger, A.; Braun, D.L.; Eich, G.; Keller, D.; Hasse, B.; et al. Large parallel screen of saliva and nasopharyngeal swabs in a test center setting proofs utility of saliva as alternate specimen for SARS-CoV-2 detection by RT-PCR. MedRxiv 2020. Available online: https:/ /www.medrxiv.org/content/10.1101/2020.12.01.202 41778v1.full (accessed on 7 April 2021).

7. FIND. SARS-CoV-2 Diagnostic Pipeline. Available online: https://www.finddx.org/covid-19/pipeline/?avance=all\&type= Rapid+diagnostic+tests\&test_target=Antigen\&status=all\&section=show-all\&action=default (accessed on 25 January 2021). 
8. Caruana, G.; Croxatto, A.; Coste, A.T.; Opota, O.; Lamoth, F.; Jaton, K.; Greub, G. Diagnostic strategies for SARS-CoV-2 infection and interpretation of microbiological results. Clin. Microbiol. Infect. 2020, 26, 1178-1182. [CrossRef]

9. Teo, A.K.J.; Choudhury, Y.; Tan, I.B.; Cher, C.Y.; Chew, S.H.; Wan, Z.Y.; Cheng, L.T.E.; Oon, L.L.E.; Tan, M.H.; Chan, K.S.; et al. Saliva is more sensitive than nasopharyngeal or nasal swabs for diagnosis of asymptomatic and mild COVID-19 infection. Sci. Rep. 2021, 11, 1-8. [CrossRef]

10. Skolimowska, K.; Rayment, M.; Jones, R.; Madona, P.; Moore, L.S.P.; Randell, P. Non-invasive saliva specimens for the diagnosis of COVID-19: Caution in mild outpatient cohorts with low prevalence. Clin. Microbiol. Infect. 2020, 12, 1711-1713. [CrossRef]

11. Berenger, B.M.; Conly, J.M.; Fonseca, K.; Hu, J.; Louie, T.; Schneider, A.R.; Singh, T.; Stokes, W.; Ward, L.; Zelyas, W. Saliva collected in universal transport media is an effective, simple and high-volume amenable method to detect SARS-CoV-2. Clin. Microbiol. Infect. 2020, 27, 656-657. [CrossRef] [PubMed]

12. Wyllie, A.L.; Fournier, J.; Casanovas-Massana, A.; Campbell, M.; Tokuyama, M.; Vijayakumar, P.; Warren, J.L.; Geng, B.; Muenker, M.C.; Moore, A.J.; et al. Saliva or Nasopharyngeal Swab Specimens for Detection of SARS-CoV-2. N. Engl. J. Med. 2020, 383, 1283-1286. [CrossRef] [PubMed]

13. Azzi, L.; Carcano, G.; Gianfagna, F.; Grossi, P.; Gasperina, D.D.; Genoni, A.; Fasano, M.; Sessa, F.; Tettamanti, L.; Carinci, F.; et al. Saliva is a reliable tool to detect SARS-CoV-2. J. Infect. 2020, 81, e45-e50. [CrossRef] [PubMed]

14. Caruana, G.; Croxatto, A.; Kampouri, E.; Kritikos, A.; Opota, O.; Foerster, M.; Brouillet, R.; Senn, L.; Lienhard, R.; Egli, A.; et al. ImplemeNting SARS-CoV-2 Rapid antigen testing in the Emergency wArd of a Swiss univErsity hospital: The INCREASE study. Microorganisms 2021, 9, 798. [CrossRef]

15. Kirkland, P.D.; Frost, M.J. The impact of viral transport media on PCR assay results for the detection of nucleic acid from SARS-CoV-2. Pathology 2020, 52, 811-814. [CrossRef] [PubMed]

16. Rogers, A.A.; Baumann, R.E.; Borillo, G.A.; Kagan, R.M.; Batterman, H.J.; Galdzicka, M.M.; Marlowe, E.M. Evaluation of Transport Media and Specimen Transport Conditions for the Detection of SARS-CoV-2 by Use of Real-Time Reverse Transcription-PCR. J. Clin. Microbiol. 2020, 58, e00708-20. [CrossRef]

17. Walsh, P.; Overmyer, C.L.; Pham, K.; Michaelson, S.; Gofman, L.; DeSalvia, L.; Tran, T.; Gonzalez, D.; Pusavat, J.; Feola, M.; et al. Comparison of respiratory virus detection rates for infants and toddlers by use of flocked swabs, saline aspirates, and saline aspirates mixed in universal transport medium for room temperature storage and shipping. J. Clin. Microbiol. 2008, 46, 2374-2376. [CrossRef] [PubMed]

18. Opota, O.; Brouillet, R.; Greub, G.; Jaton, K. Comparison of SARS-CoV-2 RT-PCR on a high-throughput molecular diagnostic platform and the cobas SARS-CoV-2 test for the diagnostic of COVID-19 on various clinical samples. Pathog. Dis. 2020, 78 , ftaa061. [CrossRef] [PubMed]

19. Berger, A.; Nsoga, M.T.N.; Perez-Rodriguez, F.J.; Aad, Y.A.; Sattonnet-Roche, P.; Gayet-Ageron, A.; Jaksic, C.; Torriani, G.; Boehm, E.; Kronig, L.; et al. Diagnostic accuracy of two commercial SARS-CoV-2 antigen-detecting rapid tests at the point of care in community-based testing centers. PLoS ONE 2021, 16, e0248921. [CrossRef]

20. Prevention CfDCa. Interim Guidelines for Collecting, Handling, and Testing Clinical Specimens from Persons for Coronavirus Disease 2019 (COVID-19). Available online: https:/ / www.cdc.gov/coronavirus/2019-ncov/lab/guidelines-clinical-specimens. html\#additional-resources (accessed on 7 April 2021).

21. Marty, F.M.; Chen, K.; Verrill, K.A. How to Obtain a Nasopharyngeal Swab Specimen. N. Engl. J. Med. 2020, 382, e76. [CrossRef] [PubMed]

22. Jacot, D.; Greub, G.; Jaton, K.; Opota, O. Viral load of SARS-CoV-2 across patients and compared to other respiratory viruses. Microbes. Infect. 2020, 22, 617-621. [CrossRef]

23. Desmet, T.; Paepe, P.; Boelens, J.; Coorevits, L.; Padalko, E.; Vandendriessche, S.; Leroux-Roels, I.; Aerssens, A.; Callens, A.; Braeckel, E.V.; et al. Combined oropharyngeal/nasal swab is equivalent to nasopharyngeal sampling for SARS-CoV-2 diagnostic PCR. BMC Microbiol. 2021, 21, 1-4. [CrossRef] [PubMed]

24. Vlek, A.L.M.; Wesselius, T.S.; Achterberg, R.; Thijsen, S.F.T. Combined throat/nasal swab sampling for SARS-CoV-2 is equivalent to nasopharyngeal sampling. Eur. J. Clin. Microbiol. Infect. Dis. 2021, 40, 193-195. [CrossRef] [PubMed]

25. Hanson, K.E.; Caliendo, A.M.; Arias, C.A.; Hayden, M.K.; Englund, J.A.; Lee, M.J.; Loeb, M.; Patel, R.; El Alayli, A.; Altayar, O.; et al. The Infectious Diseases Society of America Guidelines on the Diagnosis of COVID-19: Molecular Diagnostic Testing. Clin Infect. Dis. 2021. Available online: https:/ / www.idsociety.org/practice-guideline/covid-19-guideline-diagnostics/ (accessed on 7 April 2021).

26. Adrian Egli, R.L.; Jaton, K.; Gilbert Greub for the Swiss Society of Microbiology. Recommendation of the Swiss Society of Microbiology for usage of SARS-CoV-2 specific antigen tests. PIPETTE—SWISS LABORATORY MEDICINE NR. 6 December 2020. Available online: https://issuu.com/sulm-pipette/docs/pipette_6-2020-web (accessed on 7 April 2021).

27. Sterlin, D.; Mathian, A.; Miyara, M.; Mohr, A.; Anna, F.; Claer, L.; Quentric, P.; Fadlallah, J.; Devilliers, H.; Gorochov, G.; et al. IgA dominates the early neutralizing antibody response to SARS-CoV-2. Sci. Transl. Med. 2021, 13. Available online: https://www.science.org/doi/10.1126/scitranslmed.abd2223 (accessed on 7 April 2021). [CrossRef] [PubMed] 
28. Weitzel, T.; Legarraga, P.; Iruretagoyena, M.; Pizarro, G.; Vollrath, V.; Araos, R.; Munita, J.M.; Porte, L. Comparative evaluation of four rapid SARS-CoV-2 antigen detection tests using universal transport medium. Travel Med. Infect. Dis. 2021, $39,101942$. [CrossRef] [PubMed]

29. Furness, S.; Worthington, H.V.; Bryan, G.; Birchenough, S.; McMillan, R. Interventions for the management of dry mouth: Topical therapies. Cochrane Database Syst. Rev. 2011, 12. Available online: https://www.cochranelibrary.com/cdsr/doi/10.1002/146518 58.CD008934.pub2/full (accessed on 7 April 2021). [CrossRef] [PubMed] 